TRANSACTIONS OF THE

AMERICAN MATHEMATICAL SOCIETY

Volume 358, Number 7, Pages 2949-2964

S 0002-9947(05)03741-4

Article electronically published on May 26, 2005

\title{
ALEXANDER POLYNOMIALS OF EQUIVARIANT SLICE AND RIBBON KNOTS IN $S^{3}$
}

\author{
JAMES F. DAVIS AND SWATEE NAIK
}

\begin{abstract}
This paper gives an algebraic characterization of Alexander polynomials of equivariant ribbon knots and a factorization condition satisfied by Alexander polynomials of equivariant slice knots.
\end{abstract}

\section{INTRODUCTION}

Definition 1.1. A knot in $S^{3}$ is slice if it bounds a slice disk, i.e., a smooth 2-disk properly embedded in the 4-ball. It is called ribbon if it bounds a slice disk such that the radius function of the 4-ball, when restricted to the disk, is a Morse function with critical points of index 0 and 1 only. Such a disk is called a ribbon disk.

Ribbon knots are alternatively defined [9, 23] as knots which bound an immersed 2-disk $h: D^{2} \rightarrow S^{3}$, so that every component of self-intersection is an $\operatorname{arc} A$ so that $h^{-1}(A)$ consists of two arcs in $D^{2}$, one of which is interior. Whether or not all slice knots are ribbon is an open question which first appeared as Problem 25 in Fox's problem list [9] in 1966. Also see Problem 1.33 in [13].

Definition 1.2. A knot $K$ in $S^{3}$ is said to be periodic with period $q$ if there exists an order $q$ orientation preserving diffeomorphism $f: S^{3} \rightarrow S^{3}$ such that $f(K)=K$, and the fixed point set of $f$ is a circle disjoint from $K$.

By the positive solution to the Smith Conjecture [19], $\operatorname{Fix}(f)$ is unknotted. It follows that the quotient space of $S^{3}$ under the $\mathbf{Z} / q$ action is again $S^{3}$. We denote the image of $K$ in the quotient space by $\bar{K}$, and call it the quotient knot. We let $B=\operatorname{Fix}(f)$ and $\bar{B}$ be its image in the quotient space, and refer to them as the axes. The quotient link is $\bar{B} \cup \bar{K}$.

For example, the trefoil in its usual diagram is clearly seen to have period 3 and with an alternate diagram we see that it has period 2; both quotient knots are trivial.

Definition 1.3. A period $q$ knot is called q-equivariant slice (ribbon) if the periodic diffeomorphism of $S^{3}$ extends to that of $B^{4}$ and there is an invariant slice (ribbon) disk.

A periodic knot which bounds a 2-disk immersed in $S^{3}$ with ribbon singularities, where the 2-disk is preserved by the periodic action is equivariant ribbon (see [21]).

Received by the editors May 20, 2002 and, in revised form, May 11, 2004.

2000 Mathematics Subject Classification. Primary 57M25.

The first author was partially supported by a grant from the National Science Foundation.

(C)2005 American Mathematical Society Reverts to public domain 28 years from publication 2949 
An example of a 2-equivariant ribbon knot is the Stevedore's knot $6_{1}$; see Figure 2.2 ,

Clearly, equivariant ribbon knots are equivariant slice. In fact, all the knots that have been shown so far to be equivariant slice are equivariant ribbon.

Conjecture 1.4. An equivariant slice knot is equivariant ribbon.

It follows from Smith theory [24] that the fixed point set of the periodic map of $B^{4}$ is a disk. The fixed point disk may be knotted, as there are non-standard finite group actions on $B^{4}$ [7]. However, the quotient manifold is a homology 4ball whose boundary is $S^{3}$. In fact, one can show that the quotient manifold is a simply-connected homology 4-ball whose boundary is $S^{3}$, and hence, by topological surgery, that it is homeomorphic to a 4-ball.

It was shown in Corollary 3.3 of [21] that the linking number of the equivariant slice knot with its axis is always 1 . Further obstructions to a slice, periodic knot being equivariant slice, were obtained in [3, 15, 21] in terms of Seifert matrices, metabolizers of Seifert forms, Casson-Gordon invariants, and surgery on the quotient knot. In [3, 15] examples were given of knots which are slice, periodic, and have linking number one with the axis, but cannot be equivariant slice. (See also Example 2.5.)

The goal of this paper is to characterize Alexander polynomials of equivariant ribbon knots and to give a necessary condition that an Alexander polynomial of an equivariant slice knot must satisfy. Our results place restrictions on which knots can be equivariant slice/ribbon and construct new examples of equivariant ribbon knots.

Alexander polynomials of knots, of slice knots, and of periodic knots with linking number 1 have been characterized, and before we state our results we review this earlier work. We first make a convention: all polynomials in the Laurent polynomial $\operatorname{ring} \mathbf{Z}\left[t, t^{-1}\right]$ are only defined up to multiplication by $\pm t^{i}$. Similarly, a two variable polynomial in $\mathbf{Z}[\mathbf{Z} \times \mathbf{Z}]=\mathbf{Z}\left[g, g^{-1}, t, t^{-1}\right]$ or in $\mathbf{Z}[\mathbf{Z} / q \times \mathbf{Z}]=\mathbf{Z}\left[g, t, t^{-1}\right] /\left\langle g^{q}\right\rangle$ is only defined up to multiplication by $\pm g^{i} t^{j}$. All equalities involving polynomials will only hold up to such multiples.

Definition 1.5. A polynomial $\Delta(t) \in \mathbf{Z}\left[t, t^{-1}\right]$ is an (abstract) Alexander polynomial if

(1) $\Delta(1)=1$,

(2) $\Delta(t)=\Delta\left(t^{-1}\right)$.

Proposition 1.6 (Seifert, see [16]). An Alexander polynomial of a knot is an abstract Alexander polynomial. If $\Delta(t)$ is an abstract Alexander polynomial, then there is a knot whose Alexander polynomial is $\Delta(t)$.

The corresponding result for slice and ribbon knots is stated next. Proposition 1.7 below combines the result of Fox [8, 10] regarding factorization of the Alexander polynomial of a slice knot together with a result of Terasaka 26] that, given a polynomial satisfying the Fox factorization condition, there exists a slice, in fact, a ribbon knot with the polynomial as its Alexander polynomial (see also Section 3).

Proposition 1.7. If $\Delta(t)$ is the Alexander polynomial of a slice knot, then there is a polynomial $p(t) \in \mathbf{Z}\left[t, t^{-1}\right]$ so that $\Delta(t)=p(t) p\left(t^{-1}\right)$. Conversely, given a polynomial $p(t) \in \mathbf{Z}\left[t, t^{-1}\right]$ with $p(1)=1$, there exists a ribbon knot whose Alexander polynomial is $p(t) p\left(t^{-1}\right)$. 
Alexander polynomials of periodic knots which have linking number one with the axis have been characterized. Proposition 1.8 below states the result of K. Murasugi 20] regarding the factorization of the Alexander polynomial of a periodic knot, together with the existence result from [6].

Proposition 1.8. Let $K$ be a period $q$ knot with linking number one. Let $\Delta_{K}(t)$ and $\Delta_{\bar{K}}(t)$ be the Alexander polynomials of $K$ and the quotient knot $\bar{K}$ respectively. Then there exists a polynomial $\Delta_{\mathbf{Z} / q}(g, t) \in \mathbf{Z}[\mathbf{Z} / q \times \mathbf{Z}]$ such that

(1) $\Delta_{\bar{K}}$ divides $\Delta_{K}$,

(2) $\Delta_{K}(t) / \Delta_{\bar{K}}(t)=\prod_{i=1}^{q-1} \Delta_{\mathbf{Z} / q}\left(\zeta^{i}, t\right)$, where $\zeta$ is a primitive $q$-th root of unity,

(3) $\Delta_{\mathbf{Z} / q}(g, t)=\Delta_{\mathbf{Z} / q}\left(g^{-1}, t^{-1}\right)$,

(4) $\Delta_{\mathbf{Z} / q}(g, 1)=1$,

(5) $\Delta_{\mathbf{Z} / q}(1, t)=\Delta_{\bar{K}}(t)$.

Conversely, given a polynomial $\Delta_{\mathbf{Z} / q}(g, t) \in \mathbf{Z}[\mathbf{Z} / q \times \mathbf{Z}]$ satisfying conditions (3) and (4) above, there is a period $q$ knot with linking number one, with the Alexander polynomial of the quotient knot given by (5), and the Alexander polynomial of the periodic knot given by (2).

For a periodic knot, the polynomial $\Delta_{\mathbf{Z} / q}(g, t)$ is the image of the 2-variable Alexander polynomial of the quotient link under the map $\mathbf{Z}[\mathbf{Z} \times \mathbf{Z}] \rightarrow \mathbf{Z}[\mathbf{Z} / q \times \mathbf{Z}]$. See [20, 6]. We will call $\Delta_{\mathbf{Z} / q}(g, t)$ the Murasugi polynomial of the periodic knot.

A consequence of the fact that a ribbon disk has only critical points of index 0 and 1 is that the exterior $X$ of a ribbon disk is $B^{4}$ with 1-handles and 2-handles attached. Clearly, $X$ has the homotopy type of a 2-complex; a homological consequence is that the infinite cyclic cover $\widetilde{X}$ of $X$ has trivial $H_{2}$. (Since $X$ is a 2-complex, $H_{2}(\widetilde{X})$ is torsion-free over $\mathbf{Z}\left[t, t^{-1}\right]$, whereas the Wang sequence of the infinite cyclic cover shows that $H_{2}(\tilde{X})$ must be torsion over $\mathbf{Z}\left[t, t^{-1}\right]$.) This need not be the case if $X$ was merely the exterior of a slice disk. This homological difference between slice disks and ribbon disks is reflected in our equivariant results given below.

In Section 5, we prove the following condition on Alexander polynomials of equivariant slice knots using an argument based on Reidemeister torsion.

Theorem 1.9. Let $K$ be a q-equivariant slice knot with Murasugi polynomial $\Delta_{\mathbf{Z} / q}(g, t)$. Then there exist nonzero polynomials $a(g, t)$ and $b(g, t)$ in $\mathbf{Z}[\mathbf{Z} / q \times \mathbf{Z}]$ such that

(1) $\Delta_{\mathbf{Z} / q}(g, t) b(g, t) b\left(g^{-1}, t^{-1}\right)=a(g, t) a\left(g^{-1}, t^{-1}\right)$,

(2) $a(g, 1)=1=b(g, 1)$.

The fact that $H_{2}$ of the infinite cyclic cover of the exterior of a ribbon disk is trivial enables us to show that if $K$ is equivariant ribbon, then $b(g, t)=1$. We show that this characterizes Alexander polynomials of equivariant ribbon knots. Our result is stated below. The proof uses a handle-theoretic construction which is described in Section 3

Theorem 1.10. Let $K$ be a q-equivariant ribbon knot with Murasugi polynomial $\Delta_{\mathbf{Z} / q}(g, t)$. Then there exists a polynomial $a(g, t)$ in $\mathbf{Z}[\mathbf{Z} / q \times \mathbf{Z}]$ such that

(1) $\Delta_{\mathbf{Z} / q}(g, t)=a(g, t) a\left(g^{-1}, t^{-1}\right)$, and

(2) $a(g, 1)=1$. 
Conversely, given a polynomial $a(g, t) \in \mathbf{Z}[\mathbf{Z} / q \times \mathbf{Z}]$ with $a(g, 1)=1$, there is a q-equivariant ribbon knot whose Murasugi polynomial is given by (1), and hence whose Alexander polynomial is given by $\prod_{i=0}^{q-1} \Delta_{\mathbf{Z} / q}\left(\zeta^{i}, t\right)$.

It follows from this result that in the examples of [3, 15, the knots have Alexander polynomials of equivariant ribbon knots.

We state below a conjecture weaker than Conjecture 1.4.

Conjecture 1.11 (1). Alexander polynomials of equivariant slice knots satisfy the factorization condition in 1.9 with $b(g, t)=1$.

In Section 3, given a polynomial which satisfies the factorization conditions in Theorem 1.10, we describe a construction of a $q$-equivariant ribbon knot with that as its polynomial. A special case of our result is the known fact that given a polynomial of the form $p(t) p\left(t^{-1}\right)$, there exists a ribbon knot with that as its polynomial. Our proof is handle-theoretic and avoids the long computations of [26]. (Handletheoretic constructions of slice knots were given earlier in 11.) In Section 5 we give an argument based on Reidemeister torsion to obtain necessary factorization conditions for Alexander polynomials of equivariant slice knots and those of equivariant ribbon knots.

\section{EXAMPLES AND APPLICATIONS}

Example 2.1. The Stevedore's knot $6_{1}$ pictured in Figure 2.2 is 2-equivariant ribbon.

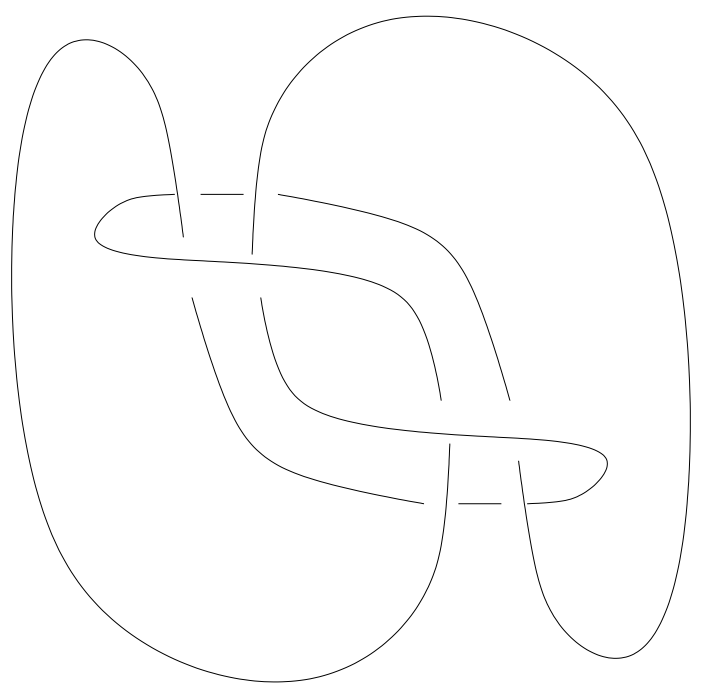

FIGURE 2.2.

\footnotetext{
${ }^{1} \mathrm{~J}$. Hillman sent us a note 12 giving partial results on this conjecture. After our paper was accepted, J. C. Cha sent us a proof [4] of our conjecture, and hence completed the characterization of Alexander polynomials of equivariant slice knots. The Reidemeister torsion techniques of our paper also lead to a proof of Cha's result, provided one works in $\mathbf{Z}[\mathbf{Z} \times \mathbf{Z}]$ and applies Lemma 2.4
} 
Here are the various polynomial invariants for Stevedore's knot:

$$
\begin{aligned}
\Delta_{K}(t) & =-2 t+5-2 t^{-1}, \\
p(t) & =-2 t+1, \\
\Delta_{\bar{K}}(t) & =1, \\
\Delta_{\mathbf{Z} / 2}(g, t) & =(g-1) t+3-2 g+\left(g^{-1}-1\right) t^{-1}, \\
a(g, t) & =(g-1) t+1 .
\end{aligned}
$$

The following is an immediate consequence of Theorem 1.9 ,

Corollary 2.3. Let $K$ be a q-equivariant slice knot with $q$ a prime. Let $\zeta$ be a primitive $q$-th root of unity. Then $\Delta_{K}(t) / \Delta_{\bar{K}}(t)$ factors into $q-1$ conjugate factors over $\mathbf{Z}[\zeta]$ each of which is of the form $\left(f_{1}(t) f_{1}\left(t^{-1}\right)\right) /\left(f_{2}(t) f_{2}\left(t^{-1}\right)\right)$, where $f_{1}(t), f_{2}(t) \in \mathbf{Z}[\zeta][t]$.

In the cases where $Z[\zeta]$ is a unique factorization domain, this can be simplified using the following lemma.

Lemma 2.4. Let $R$ be a unique factorization domain. If

$$
f(t) b(t) b\left(t^{-1}\right)=a(t) a\left(t^{-1}\right) \in R\left[t, t^{-1}\right]
$$

where $b(t)$ is nonzero, then there is a $c(t) \in R\left[t, t^{-1}\right]$ so that

$$
f(t)=c(t) c\left(t^{-1}\right) \in R\left[t, t^{-1}\right] .
$$

Proof. Let $b(t)=p_{1}(t)^{e_{1}} \cdots p_{r}(t)^{e_{r}}$ be the prime factorization of $b(t)$. Let $f_{i}$ be the largest integer so that $f_{i} \leq e_{i}$ and $p_{i}(t)^{f_{i}}$ divides $a(t)$. Let

$$
c(t)=\frac{a(t)}{\prod_{i=1}^{r} p_{i}(t)^{f_{i}} p_{i}\left(t^{-1}\right)^{e_{i}-f_{i}}} .
$$

Example 2.5. The knot $K=10_{123}$ is slice [5] and has Alexander polynomial $\Delta_{K}(t)=\left(t^{4}-3 t^{3}+3 t^{2}-3 t+1\right)^{2}$; we will show that this is not the Alexander polynomial of a 2-equivariant slice knot. If $K$ had period 2 with linking number 1 , then by considering conditions (2) and (5) of Proposition 1.8 modulo two (see also Murasugi [20]), $\Delta_{K}(t) \equiv \Delta_{\bar{K}}(t)^{2}(\bmod 2)$, and hence $\Delta_{\bar{K}}(t)=t^{4}-3 t^{3}+3 t^{2}-3 t+1$. But $\Delta_{K}(t) / \Delta_{\bar{K}}(t)=t^{4}-3 t^{3}+3 t^{2}-3 t+1$ is irreducible, and hence by Corollary 2.3 and Lemma 2.4 that $\Delta_{K}(t)$ is not an Alexander polynomial of a 2-equivariant slice knot.

It is easy to show that $\Delta_{K}(t)$ is the Alexander polynomial of a period 2 knot. This knot $K=10_{123}$ is listed as period 2 in [2, but in an email communication with the second author Jeff Weeks has told us that according to Snappea, $10_{123}$ does not have period 2 .

Here is a realization result as a consequence of Theorem 1.10 (Compare Corollary 1.2 of [6].)

Corollary 2.6. If an Alexander polynomial $\Delta(t)$ factors as $p(t) p\left(t^{-1}\right)$ with $p(t) \equiv$ $1 \bmod q$, then there exists a q-equivariant ribbon knot $K$ such that $\Delta(t)$ is the polynomial of $K$ as well as that of the quotient knot.

Proof. Write $p(t)=q \cdot h(t)+1$. Now let $a(g, t)=\left(1+g+\cdots+g^{q-1}\right) \cdot h(t)+1$, and apply Theorem 1.10 . 
Note that if $q$ is prime, $(\mathbf{Z} / q)[t]$ is a unique factorization domain, so that if $\Delta(t)=p(t) p\left(t^{-1}\right)$ and if $\Delta(t) \equiv 1 \bmod q$, then it is automatic that $p(t) \equiv 1 \bmod q$.

In 21] a list of periodic, slice knots, which are not equivariant slice, was given. There were some cases of knots with less than or equal to 10 crossings that the techniques in 21] were unable to settle. It follows from Corollary 2.6 that the Alexander polynomial of each of these knots (except $10_{123}$ ) is that of an equivariant ribbon knot.

Referring to the list of knot concordances given in [5], Examples 2.7 and 2.5 above, together with Examples 2.5 and 3.4 in 21] list all the candidates for equivariant slice knots from the tables of knots with 10 or fewer crossings.

Example 2.7. The knots $8_{8}, 9_{46}, 10_{22}$, and $10_{35}$ are all slice and have period 2 with $\lambda=1$. For each of these knots $\Delta(t) \equiv 1 \bmod 2$. Hence by Corollary 2.6 it follows that each of their Alexander polynomials is the Alexander polynomial of a 2-equivariant ribbon knot. It was shown in [15], using a characterization of the Seifert matrix, that $8_{8}$ is not 2 -equivariant slice. It is not yet known whether or not any of the others are 2-equivariant slice.

Example 2.8. In 3 an example is given of a knot which is period 2, slice, but not 2 -equivariant slice. The polynomial of this knot is $-2 t+5-2 t^{-1}$ which is $1 \bmod 2$, and hence there exists a 2 -equivariant ribbon knot with this polynomial. (See also Figure 2.2.)

\section{Construction of EQUivariant RIBBOn KNOtS}

In this section, we will prove the realization part of Theorem 1.10, For any $a(g, t) \in \mathbf{Z}[\mathbf{Z} / q \times \mathbf{Z}]$ with $a(g, 1)=1$, we construct a $q$-equivariant ribbon knot $K$ with Murasugi polynomial $a(g, t) a\left(g^{-1}, t^{-1}\right)$. We do this by constructing the quotient link $\bar{B} \cup \bar{K}$.

Remark 3.1. Let $W$ be a 4 -manifold with boundary. By attaching a $j$-handle to $W$ (see [14, VI.6]), one means constructing the manifold

$$
W^{\prime}=W \cup_{\phi} D^{j} \times D^{4-j}
$$

where $\phi: S^{j-1} \times D^{4-j} \hookrightarrow \partial W$ is an embedding. A smooth structure on $W$ leads, by "rounding corners", to a smooth structure on $W^{\prime}$, which is invariant under isotopy of $\phi$. To obtain $\partial W^{\prime}$ from $\partial W$ one proceeds by the process of surgery, i.e.,

$$
\partial W^{\prime} \cong\left(\partial W-\phi\left(S^{j-1} \times \operatorname{int} D^{4-j}\right)\right) \cup_{S^{j-1} \times S^{3-j}} D^{j} \times S^{3-j} .
$$

If one attaches a 1-handle and then a 2-handle

$$
W^{\prime \prime}=\left(W \cup_{\phi_{1}} D^{1} \times D^{3}\right) \cup_{\phi_{2}} D^{2} \times D^{2},
$$

so that the belt sphere $0 \times S^{2}$ of the 1-handle intersects the attaching sphere $S^{1} \times 0$ of the 2-handle exactly once, transversely in $\partial W^{\prime}$, then $W$ and $W^{\prime \prime}$ are diffeomorphic (see [14, VI.7]).

If $K$ is a slice knot in $S^{3}$ (e.g. the unknot), and if one attaches a cancelling pair of handles as above to $B^{4}$ whose attaching maps $\phi_{1}, \phi_{2}$ have image disjoint from $K$, then $K \subset \partial W^{\prime \prime} \cong S^{3}$ is still a slice knot, however, possibly a different knot from the original knot $K \subset S^{3}$. 
Remark 3.2. Let $D_{\bar{B}}$ and $D_{\bar{K}}$ be a pair of linear, orthogonal 2-disks in $B^{4}$. Then

$$
\bar{B} \cup \bar{K}=\left(D_{\bar{B}} \cup D_{\bar{K}}\right) \cap S^{3} \subset S^{3}
$$

is the Hopf link. Suppose one attaches a cancelling pair of handles $\phi_{1}, \phi_{2}$ as above to $B^{4}$, with $\bar{K}$ disjoint from the handles. Suppose, furthermore, that $\bar{B}$ bounds a 2-disk in $S^{3}$, disjoint from the handles. Let $B, K, D_{B}$, and $D_{K}$ be the inverse images of $\bar{B}, \bar{K}, D_{\bar{B}}, D_{\bar{K}}$ under the $q$-fold branched cover $\pi: \widehat{W^{\prime \prime}} \rightarrow W^{\prime \prime}$ branched over the unknotted 2-disk $D_{\bar{B}}$. This gives us a $q$-equivariant slice (in fact, ribbon) $\operatorname{knot} K \subset \widehat{\partial W^{\prime \prime}} \cong S^{3}$.

We will use Akbulut's modification of Kirby diagrams (see [11, Chapter 5]). A pair of cancelling handles is indicated by a 2-component link with one component marked with a dot and bounding a 2-disk (usually not explicitly drawn), which intersects the other component transversely once. The other component is equipped with an integer $k$. The component with the dot corresponds to adding a 1-handle; the second component corresponds to adding the 2-handle, with the $k$ indicating the framing. An $\operatorname{arc} A$ of the undotted component intersecting the spanning disk for the dotted component indicates that a piece of the attaching map of the 2-handle goes across the boundary of the 1-handle, i.e., $A \times D^{2} \subset D^{1} \times S^{2} \subset D^{1} \times D^{3}$.

We illustrate our construction of the quotient link through a simple example.

Example 3.3. Let $a(g, t)=1-g+g t$. Figure 3.4 indicates the $\operatorname{link} \bar{B} \cup \bar{K} \subset S^{3}$ and a pair of a 1-handle and a 2-handle attached to the 4-ball along $S^{3}$.

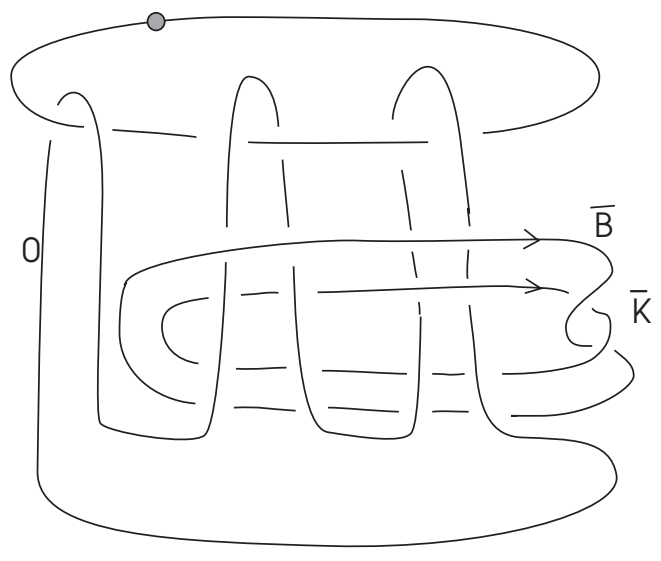

FIGURE 3.4.

Remark 3.5. Let $S_{1}$ be the circle labelled with 0 , and let $S_{2}$ be the circle labelled with the dot.

(1) There is a disk $D$ with boundary $S_{2}$, so that $S_{1}$ intersects $D$ exactly once, transversely.

(2) $\bar{B} \cup \bar{K}$ is the Hopf link and is disjoint from $S_{1} \cup S_{2}$.

(3) After erasing $\bar{K}, \bar{B}$ bounds a 2-disk in the complement of the rest of the link and $D$.

(4) $S_{1}$ is contained in a ball disjoint from $\bar{B} \cup \bar{K}$. $S_{2}$ is contained in a ball disjoint from $\bar{B} \cup \bar{K}$. 
As explained in Remark 3.2. (1), (2), and (3) imply that the $q$-fold branched cover, branched over $\bar{B}$, gives a $q$-equivariant slice knot. The complement of the slice disk is constructed from $B^{4}$ by using only 1- and 2-handles. This shows that the knot is homotopy ribbon. To see that it is ribbon, examine Figures 3.8 and 3.9 below. Item (4) is convenient for doing computations by lifting handles to the infinite cyclic cover of the knot complement.

What remains in this example is the computation of the Murasugi polynomial. This can be accomplished in three ways: by lifting handles (this is what we do next), by using Poincaré-Lefschetz duality and Reidemeister torsion (see Section 5), or by direct analysis of Figure 3.9 below.

Let $\pi: X \rightarrow \bar{X}$ be the $q$-fold branched cover of $\bar{X}=S^{3}-\bar{K}$, branched over $\bar{B}$. Thus $X=S^{3}-K$. Let $\widetilde{X} \rightarrow X$ be the infinite cyclic cover of $X$. Since the action of $\mathbf{Z} / q$ on $X$ has a nonempty fixed set, the action lifts to $\tilde{X}$. Then $\mathbf{Z} / q \times \mathbf{Z}$ acts on $\widetilde{X}$ with quotient $\bar{X}$. Let $q: \widetilde{X} \rightarrow \bar{X}$ be the quotient map. Note that before the handles are added, $\widetilde{X}=\mathbf{R} \times \mathbf{R}^{2}$.

Let $S_{1}$ and $S_{2}$ be the closed curves indicating the 1- and 2-handles respectively. Then $q^{-1}\left(S_{1}\right)$ and $q^{-1}\left(S_{2}\right)$ are disjoint collections of closed curves, freely and transitively permuted by $\mathbf{Z} / q \times \mathbf{Z}$.

Definition 3.6. The equivariant linking of $S_{1}$ and $S_{2}$,

$$
\operatorname{lk}_{\mathbf{Z} / q \times \mathbf{Z}}\left(S_{1}, S_{2}\right)=\sum_{i, j \in \mathbf{Z}} \operatorname{lk}\left(\widetilde{S}_{1}, g^{i} t^{j} \widetilde{S}_{2}\right) g^{i} t^{j} \in \mathbf{Z}[\mathbf{Z} / q \times \mathbf{Z}],
$$

is defined by choosing a component $\widetilde{S}_{i}$ of $q^{-1}\left(S_{i}\right)$ and an orientation for that component. The equivariant linking is well defined up to multiples of $\pm g^{i} t^{j}$.

If $-: \mathbf{Z}[\mathbf{Z} / q \times \mathbf{Z}] \rightarrow \mathbf{Z}[\mathbf{Z} / q \times \mathbf{Z}]$ is the standard involution

$$
\overline{\sum a_{i, j} g^{i} t^{j}}=\sum a_{i, j} g^{-i} t^{-j}
$$

then the equivariant linking form satisfies the symmetry condition

$$
\mathrm{lk}_{\mathbf{Z} / q \times \mathbf{Z}}\left(S_{2}, S_{1}\right)=\overline{\mathrm{lk}_{\mathbf{Z} / q \times \mathbf{Z}}\left(S_{1}, S_{2}\right)} .
$$

The equivariant linking can be computed from a diagram such as Figure 3.4 as follows. First choose orientations of $S_{1}$ and $S_{2}$ and then choose a point $P_{0} \in S_{1}$ corresponding to an overcrossing of $S_{1}$ (over $S_{2}$ ). Then

$$
\mathrm{lk}_{\mathbf{Z} / q \times \mathbf{Z}}\left(S_{1}, S_{2}\right)=\sum_{P} \varepsilon_{P} g^{\operatorname{lk}\left(C_{P}, \bar{B}\right)} t^{\operatorname{lk}\left(C_{P}, \bar{K}\right)}
$$

where the sum is over all points where $S_{1}$ crosses over $S_{2}$. Here $\varepsilon_{P}= \pm 1$ where the sign corresponds to whether the crossing is positive or negative. Here $C_{P}$ is the curve which starts at $P_{0}$, travels along $S_{1}$ following the orientation until it reaches $P$ (which is at an overcrossing), switches down to $S_{2}$, follows $S_{2}$ against the orientation until reaching the point under $P_{0}$, and then jumps up to $P_{0}$. The reader should now study Figure 3.4 to check that the equivariant linking is $1-g+g t$. The proof that the equivariant linking can be computed in this way is similar to the corresponding result for the classical linking number [23].

Theorem 3.7. Suppose one has a 4-component link satisfying the conditions of Remark 3.5. Let a $(g, t)=\mathrm{k}_{\mathbf{Z} / q \times \mathbf{Z}}\left(S_{1}, S_{2}\right)$. Then the Murasugi polynomial of the corresponding q-equivariant ribbon knot is a $(g, t) a\left(g^{-1}, t^{-1}\right)$. 
Proof. The boundary of the 4-manifold given by adding the 1-handle along $S_{1}$ and the 2-handle along $S_{2}$ is the same as the 3-manifold given by doing 0-framed surgeries along the curves $S_{1}$ and $S_{2}$ (see [11 pp. 171-172]). The advantage of doing this is that the first homology of the 3-manifold is presented by the linking matrix of the surgery curves (see [11, p. 165]). This all works equivariantly, and one sees that if $\partial W^{\prime \prime}$ denotes the result of the surgeries and $\widetilde{X}^{\prime \prime}$ denotes the infinite cyclic cover of the complement of the equivariant knot, then

$$
H_{1}\left(\tilde{X}^{\prime \prime}\right) \cong \operatorname{cok}\left(\left(\begin{array}{cc}
0 & a(g, t) \\
a\left(g^{-1}, t^{-1}\right) & *
\end{array}\right): \mathbf{Z}[\mathbf{Z} / q \times \mathbf{Z}]^{2} \rightarrow \mathbf{Z}[\mathbf{Z} / q \times \mathbf{Z}]^{2}\right) .
$$

The first homology remains unchanged after removing the inverse image of the branch set. The Murasugi polynomial is thus the determinant of the above matrix.

We now return to our example (Figure 3.4), and draw a picture of the resulting ribbon knot.

By isotopy the picture in Figure 3.4 can be changed to the picture in Figure 3.8.

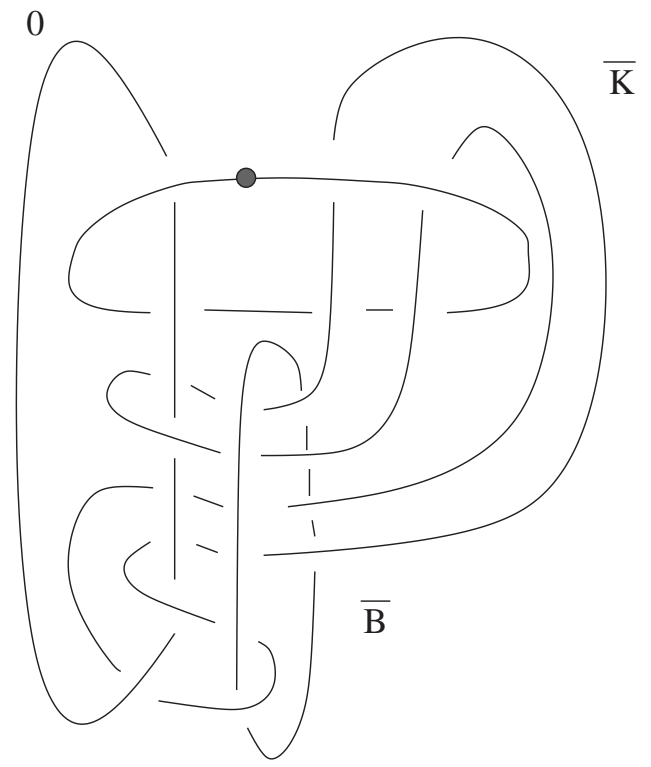

FiguRE 3.8 .

In order to see the image of $\bar{K}$ and $\bar{B}$ in the boundary of the new $B^{4}$, eliminate the intersections of $\bar{K}$ with the horizontal disk $D$ by sliding $\bar{K}$ over the 2 -handle and then erase the canceling pair of the 1-handle and the 2-handle. We see the result in Figure 3.9.

It is clear from Figure 3.9 that in the $q$-fold cyclic cover of $S^{3}$ branched over $\bar{B}$ the knot $\bar{K}$ will lift to a $q$-equivariant ribbon knot.

Our proof of the realization half of Theorem 1.10 is related to Section 2 of [6] where a construction was given to obtain a periodic knot with desired Alexander polynomial. 


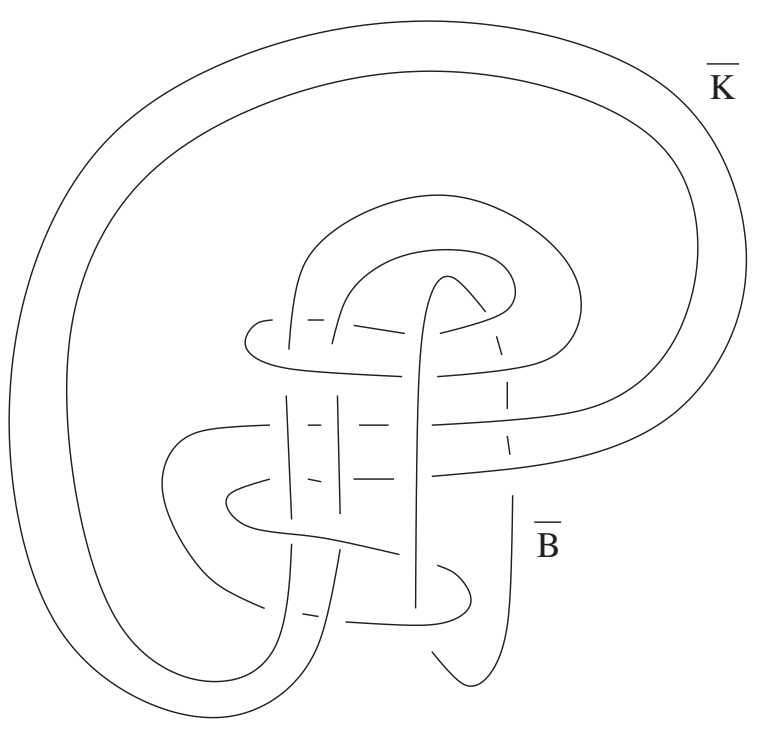

FIGURE 3.9 .

Theorem 3.10. Given a polynomial $a(g, t) \in \mathbf{Z}[\mathbf{Z} / q \times \mathbf{Z}]$ with $a(g, 1)=1$, there is a q-equivariant ribbon knot whose Murasugi polynomial is a $(\mathrm{g}, t) a\left(g^{-1}, t^{-1}\right)$.

Proof. By Theorem 3.7, we must find a 4-component link $S_{1} \cup S_{2} \cup \bar{B} \cup \bar{K}$ satisfying the conditions of Remark 3.5 so that $\operatorname{lk}_{\mathbf{Z} / q \times \mathbf{Z}}\left(S_{1}, S_{2}\right)=a(g, t)$.

Let $a(g, t)=1+\sum_{i=0}^{q-1} h_{i}(t) g^{i}$, where $h_{i}(t)=\sum_{j} a_{i, j} t^{j}$. Since $a(g, 1)=1$, $\sum_{j} a_{i, j}=0$ for all $i$. In Example 3.3 we had $a(g, t)=1+h_{0}(t)+g h_{1}(t), h_{0}(t)=0$, and $h_{1}(t)=-1+t$.

The desired link is indicated by Figures 3.11 and 3.12 The polynomial $h_{i}(t)$ determines what goes in Box $i$ below.

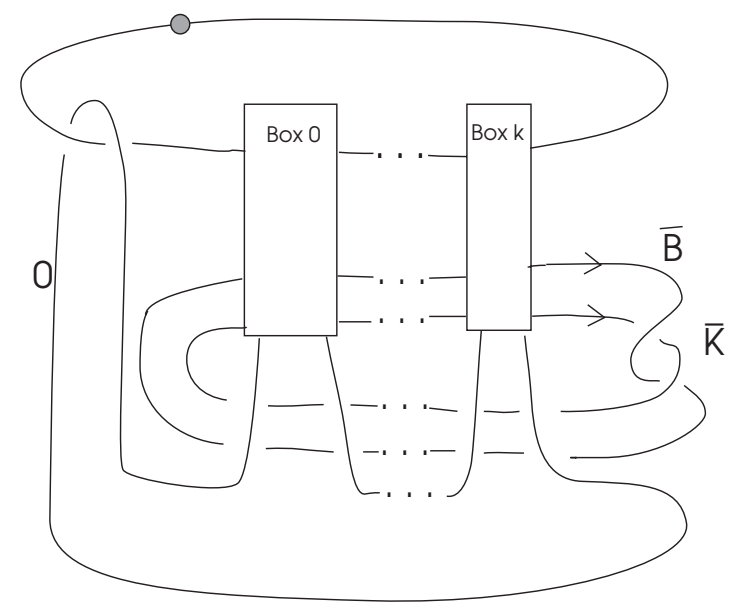

FiguRE 3.11. 
Figure 3.12 illustrates Box 2 where $h_{2}(t)=3 t^{3}-t^{2}-2 t^{-1}$.

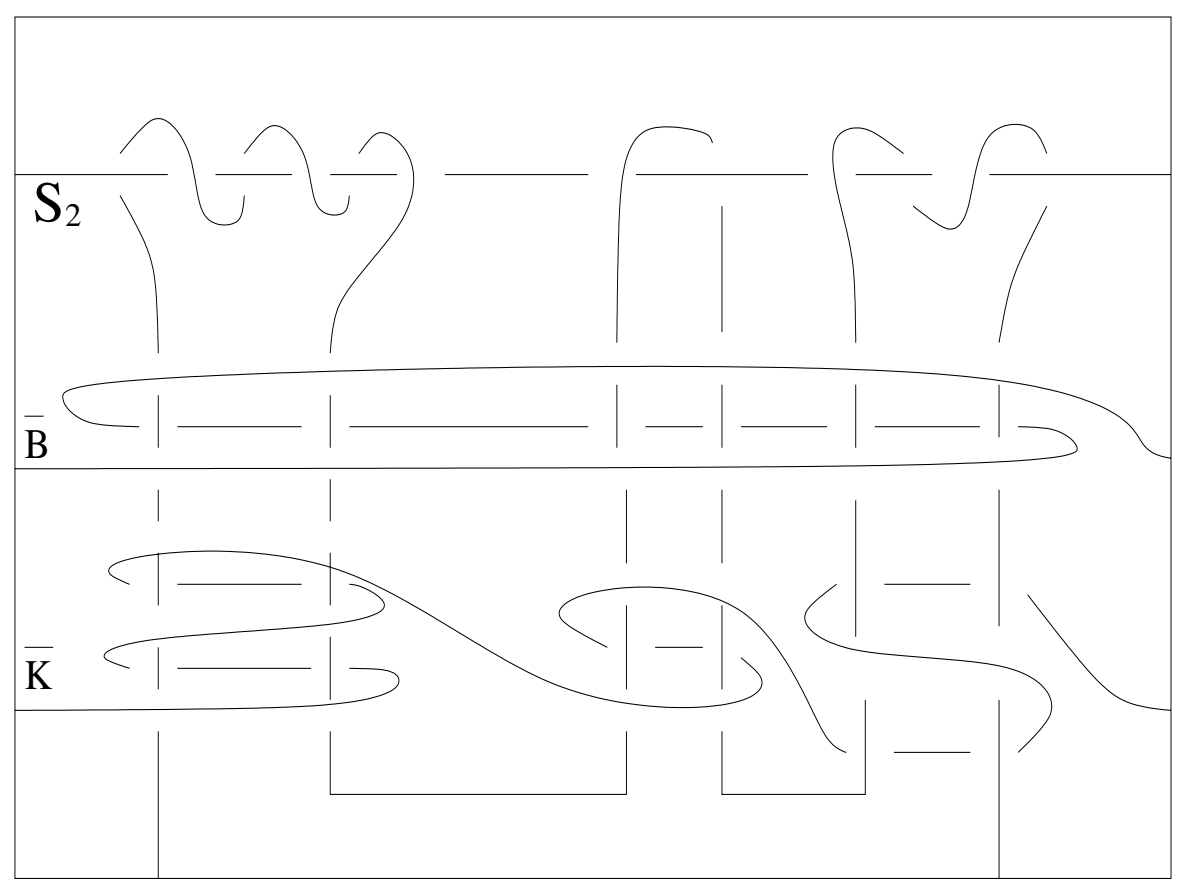

FiguRE 3.12.

Here $a_{2,4}=3$ determines the shape of the "cactus" on the left; the 2 determines the linking with $\bar{B}$, the 4 determines the linking with $\bar{K}$ and the 3 determines the linking with $S_{1}$. With this construction $\operatorname{lk}_{\mathbf{z}_{q} \times \mathbf{Z}}\left(S_{1}, S_{2}\right)=a(g, t)$. Since $\sum_{j} a_{i, j}=0$ for all $i, \bar{B}$ bounds a disk (below the boxes) in the complement of $S_{1} \cup S_{2}$. The other conditions of Remark 3.5 are clearly satisfied.

The quotient knot is obtained by adding a 1-handle along $S_{1}$ and a 2-handle along $S_{2}$. By sliding strands over the 2-handle, Figure 3.11 can be transformed into one similar to Figure 3.9 showing the quotient knot to be ribbon. The desired $q$-equivariant ribbon knot is obtained by taking the $q$-fold cover of $S^{3}$ branched over $\bar{B}$.

\section{BACKGRound on REIDEMEISTER tORSION}

Call a chain complex $C$ over a ring $A$ finite if $\bigoplus C_{i}$ is a finitely generated module, free if each $C_{i}$ is a free module, based if each $C_{i}$ is a free module equipped with a basis, and acyclic if each $H_{i}(C)$ is zero. For a finite, based, acyclic complex $C$ over a ring $A$, one can define $\left[18\right.$ the torsion $\tau(C) \in \widetilde{K}_{1}(A)$, where $\widetilde{K}_{1}(A)=K_{1}(A) /\{ \pm I\}$, $K_{1}(A)=G L(A) /[G L(A), G L(A)]$, and $G L(A)=\bigcup_{n=1}^{\infty} G L_{n}(A)$, where $G L_{n}(A) \hookrightarrow$ $G L_{n+1}(A)$ via $M \mapsto\left(\begin{array}{cc}M & 0 \\ 0 & 1\end{array}\right)$. When $A$ is a commutative ring, there is a determinant homomorphism

$$
K_{1}(A) \longrightarrow A^{\times}=\{a \in A: a b=1 \text { for some } b \in A\},
$$


and so in the commutative case the torsion defines an element in the units of $A$ modulo $\{ \pm 1\}$. In this paper, we only use the case of $A$ commutative and we only use the image of $\tau(C)$ in $A^{\times}$, so the reader who is queasy about algebraic $K$-theory may replace $K_{1}(A)$ by $A^{\times}$if so desired.

When $R \rightarrow A$ is a ring homomorphism and $C$ is a finite, free chain complex over $R$ so that $A \otimes_{R} C$ is acyclic, one can define the algebraic Reidemeister torsion

$$
\Delta(C ; A) \in \widetilde{K}_{1}(A) / \widetilde{K}_{1}(R)
$$

by assigning any $R$-basis to $C$ and defining $\Delta(C ; A)$ to be the image of $\tau\left(A \otimes_{R} C\right)$. Actually, in this paper, $C$ will be equipped with an equivalence class of bases, where the torsion of the change of basis sits in a subgroup $S \subset \widetilde{K}_{1}(R)$, in which case $\Delta(C ; A) \in \widetilde{K}_{1}(A) / S$ is well defined.

We shall be concerned with the following geometric situation. Let $X$ be a finite $C W$-complex equipped with a map $X \rightarrow B G$, where $B G$ is a connected CW complex with $\pi_{1}(B G)=G$ and all higher homotopy groups zero. Let $\widetilde{X}$ be the corresponding $G$-cover of $X$. Then $C(\widetilde{X})$ is a finite, free $\mathbf{Z} G$-chain complex, and by choosing a cell in $\widetilde{X}$ above each cell in $X$ and choosing an orientation for each such cell, $C(\widetilde{X})$ becomes a finite, based chain complex over $\mathbf{Z} G$. Suppose $\mathbf{Z} G \longrightarrow A$ is a map of rings and that $C(X ; A)=A \otimes_{\mathbf{z} G} C(\widetilde{X})$ is acyclic. In this case one defines the Reidemeister torsion $\Delta(X ; A) \in K_{1}(A) / \pm G$, where $\pm G$ refers to the subgroup generated by the image of the elements $\pm g \in G L_{1}(\mathbf{Z} G)$ where $g \in G$.

Let $f: M \rightarrow X$ be a cellular map of $C W$-complexes. Let $C(f ; A)$ be the algebraic mapping cone of $f_{\#}: C(M ; A) \rightarrow C(X ; A)$. If $C(f ; A)$ is acyclic, its torsion is denoted $\Delta(f ; A)$.

We need three background lemmas, which correspond to three properties of the Alexander polynomial: it is a polynomial, it is symmetric, and it augments to 1.

Lemma 4.1. Let $C$ be a finite, based chain complex over $R$ which has the chain homotopy type of a finite, free complex $D=\left\{D_{i+1} \rightarrow D_{i}\right\}$. If $R \rightarrow A$ is a ring homomorphism so that $A \otimes_{R} C$ is acyclic, then $\tau\left(A \otimes_{R} C\right)^{(-1)^{i}}$ can be represented by a matrix whose coefficients are in the image of $R$.

Proof. Give $D$ an $R$-basis. Let $h: C \rightarrow D$ be a chain homotopy equivalence and give the algebraic mapping cone $C_{i}(h)=C_{i-1} \oplus D_{i}$ an $R$-basis induced from that of $C$ and $D$. Note that $C(h)$ is acyclic, so $\tau(C(h)) \in \widetilde{K}_{1}(R)$. Finally,

$$
\tau\left(A \otimes_{R} C\right)=\operatorname{im} \tau(C(h)) \cdot \operatorname{det}\left(A \otimes_{R} D_{i+1} \rightarrow A \otimes_{R} D_{i}\right)^{(-1)^{i}} .
$$

A ring with involution is a ring $A$ equipped with a map $-: A \longrightarrow A$ satisfying $\overline{a+b}=\bar{a}+\bar{b}, \overline{a b}=\bar{b} \bar{a}, \overline{\bar{a}}=a$, and $\overline{1}=1$. An example of such is a group $\operatorname{ring} \mathbf{Z} G$, equipped with the involution $\overline{\sum a_{g} g}=\sum a_{g} g^{-1}$. Note that an involution on ring $A$ induces a homomorphism $-: K_{1}(A) \rightarrow K_{1}(A)$.

Recall that a triad $\left(X ; X_{+}, X_{-}\right)$of dimension $n$ is a compact manifold $X$ of dimension $n$, together with a decomposition of its boundary $\partial X=X_{+} \cup X_{-}$, where $X_{+}$and $X_{-}$are compact $(n-1)$-dimensional manifolds with $\partial X_{+}=X_{+} \cap X_{-}=$ $\partial X_{-}$. We allow the possibility of $X_{+}$and/or $X_{-}$being empty. A map of triads $f:\left(M ; M_{+}, M_{-}\right) \rightarrow\left(X ; X_{+}, X_{-}\right)$is a map $f: M \rightarrow X$ which restricts to maps $M_{+} \rightarrow X_{+}$and $M_{-} \rightarrow X_{-}$. 
Lemma 4.2. Let $X$ be a compact, oriented manifold of dimension n. Let $X \rightarrow B G$ be a continuous map and $\mathbf{Z} G \rightarrow A$ a map of rings with involution.

(1) If $X$ is closed and $C(X ; A)$ is acyclic, then $\Delta(X ; A)=\overline{\Delta(X ; A)}^{(-1)^{n-1}}$.

(2) If $C(X ; A)$ and $C(\partial X ; A)$ are acyclic, then

$$
\Delta(\partial X ; A)=\Delta(X ; A) \overline{\Delta(X ; A)}^{(-1)^{n}} .
$$

(3) If $f: M \rightarrow X$ is a proper, degree one map between compact manifolds which restricts to a homotopy equivalence $\partial M \rightarrow \partial X$ and if $C(f ; A)$ is acyclic, then $\Delta(f ; A)=\overline{\Delta(f ; A)}^{(-1)^{n-1}}$.

(4) If $f:\left(M ; M_{+}, M_{-}\right) \rightarrow\left(X ; X_{+}, X_{-}\right)$is a degree one map of triads, and if $\left.f\right|_{M_{-}}: M_{-} \rightarrow X_{-}$is a homotopy equivalence, then $\Delta\left(\left.f\right|_{M_{+}} ; A\right)=$ $\Delta(f ; A) \overline{\Delta(f ; A)}^{(-1)^{n}}$.

Proof. (1) The proof follows Milnor [17] and Wall [28, Chapter 2]. Define $C^{*}(X ; A)$ $=\operatorname{Hom}_{A}(C(X), A)$, and give it the dual basis. Then Poincaré duality gives a chain equivalence $C^{*}(X ; A) \rightarrow C(X ; A)$ whose mapping cone has trivial torsion (this is by the dual cell proof of Poincaré duality). It follows that the domain and range have equal torsion, and after taking into account the dual maps and dimension shift the torsion of the domain is $\overline{\Delta(X ; A)}{ }^{(-1)^{n-1}}$. (2) follows from a relative version of (1). Finally (3) and (4) follow because $C(f ; A)$ satisfies Poincaré duality since $f$ has degree one.

For a group $G$, let $\epsilon: \mathbf{Z} G \rightarrow \mathbf{Z}$ be the augmentation map $\epsilon\left(\sum a_{g} g\right)=\sum a_{g}$ and $S_{G}=\{\alpha \in \mathbf{Z} G: \epsilon(\alpha)=1\}$.

The following lemma is proven in 25 .

Lemma 4.3. Let $C$ be a finite, free chain complex over $\mathbf{Z} G$ for a finitely generated abelian group $G$. Then $\mathbf{Z} \otimes_{\mathbf{Z} G} C$ is acyclic if and only if $S_{G}^{-1} C=S_{G}^{-1} \mathbf{Z} G \otimes_{\mathbf{Z} G} C$ is acyclic.

The localization $S_{G}^{-1} \mathbf{Z} G$ is defined abstractly, but in cases of interest there are explicit realizations:

$$
\begin{gathered}
S_{\mathbf{Z}}^{-1} \mathbf{Z}[\mathbf{Z}]=\left\{f(t) / h(t) \in \mathbf{Q}(t): f, h \in \mathbf{Z}\left[t, t^{-1}\right], h(1)=1\right\}, \\
S_{\mathbf{Z} / q \times \mathbf{Z}}^{-1} \mathbf{Z}[\mathbf{Z} / q \times \mathbf{Z}]=\{f(g, t) / h(g, t) \in \mathbf{Q}[\mathbf{Z} / q] \times \mathbf{Q}(t): \\
\left.f, h \in \mathbf{Z}[\mathbf{Z} / q]\left[t, t^{-1}\right], h(1,1)=1\right\}, \\
S_{\mathbf{Z} \times \mathbf{Z}}^{-1} \mathbf{Z}[\mathbf{Z} \times \mathbf{Z}]=\left\{f(g, t) / h(g, t) \in \mathbf{Q}(g, t): f, h \in \mathbf{Z}\left[g, g^{-1}, t, t^{-1}\right], h(1,1)=1\right\} .
\end{gathered}
$$

Lemma 4.4. Let $K$ be a knot. Let $L$ be a 2-component link with linking number one.

(1) There are tubular neighborhoods $N(K)$ and $N(L)$ and proper maps $f$ : $S^{3}-N(K) \rightarrow S^{1} \times D^{2}$ and $h: S^{3}-N(L) \rightarrow S^{1} \times S^{1} \times[-1,1]$ inducing isomorphisms on homology and homeomorphisms on the respective boundaries.

(2) The chain complexes $C\left(f ; S_{\mathbf{Z}}^{-1} \mathbf{Z}[\mathbf{Z}]\right)$ and $C\left(h ; S_{\mathbf{Z} \times \mathbf{Z}}^{-1} \mathbf{Z}[\mathbf{Z} \times \mathbf{Z}]\right)$ are acyclic, $\Delta\left(f ; S_{\mathbf{Z}}^{-1} \mathbf{Z}[\mathbf{Z}]\right)=\Delta_{K}(t)$, and $\Delta\left(h ; S_{\mathbf{Z} \times \mathbf{Z}}^{-1} \mathbf{Z}[\mathbf{Z} \times \mathbf{Z}]\right)=\Delta_{L}(t)$.

(3) If $L=\bar{B} \cup \bar{K}$ is a link with $\bar{B}$ unknotted, then $\Delta\left(h ; S_{e \times \mathbf{Z}}^{-1} \mathbf{Z}[\mathbf{Z} / q \times \mathbf{Z}]\right)$ represents the Murasugi polynomial of the period $q$ knot given by taking the q-fold cover of $S^{3}$ branched over $\bar{B}$. 
Proof. (1) Let $N(K)$ be the open disk bundle of a tubular neighborhood of $K$. There is a commutative diagram

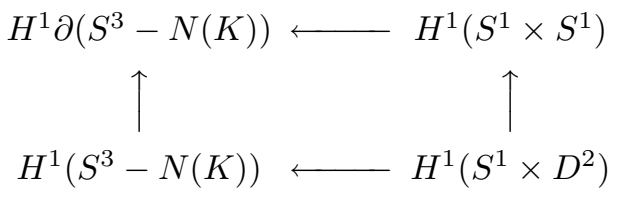

where the vertical maps are induced by inclusions of spaces, and the horizontal maps are isomorphisms given by Alexander duality. Realize the top horizontal map by a homeomorphism, and extend this to a map $f$ using that $S^{1} \times D^{2}$ is a $K(\pi, 1)$ space (see [29, V.6.9]). The construction of $h$ is similar, and linking number one is used to see that $h$ gives an isomorphism on homology.

(2) The acyclicity follows from Lemma 4.3. The relationship between Alexander polynomials and Reidemeister torsion is well known (see [17] and 27]). In our case it follows since $C(f ; \mathbf{Z}[\mathbf{Z}])$ has the chain homotopy type of a presentation of $H_{1}$ of the infinite cyclic cover, and the determinant of a presentation is the Alexander polynomial. The argument for the Alexander polynomial of the link is similar.

(3) In the statement

$$
S_{e \times \mathbf{Z}}=\{\alpha(g, t) \in \mathbf{Z}[\mathbf{Z} \times \mathbf{Z}]: \alpha(g, 1)=1\} \subset S_{\mathbf{Z} \times \mathbf{Z}} .
$$

We see $\mathbf{Z} \otimes_{\mathbf{Z}[\mathbf{Z}]} C(h ; \mathbf{Z}[\mathbf{Z} / q \times \mathbf{Z}])$ is acyclic since $\bar{B}$ is unknotted. Hence by Lemma 4.3. $S_{e \times \mathbf{Z}}^{-1} C(h ; \mathbf{Z}[\mathbf{Z} / q \times \mathbf{Z}])$ is acyclic. This result then follows from (2), since the Murasugi polynomial is represented by the Alexander polynomial of the link.

\section{Proofs of Theorems 1.9 and 1.10}

Let $K$ be a knot of period $q$ which is equivariant slice. Let $D_{K}$ be the slice disk, invariant under the group action, and let $D_{B}$ be the fixed set of the action on $D^{4}$. Let $B=D_{B} \cap S^{3}$. Then the linking number of $K$ and $B$ in $S^{3}$ is one 21. (The basic idea is that the linking number equals the intersection number of $D_{K}$ and $D_{B}$. Intersection points correspond to fixed points in $D_{K}$, and there can be only one.) Let $\bar{B}^{4}=B^{4} /(\mathbf{Z} / q)$; this is a topological ball with boundary $S^{3}$. Let $D_{\bar{B}} \subset \bar{B}^{4}$ denote the image of the fixed set in the quotient, $D_{\bar{K}} \subset \bar{B}^{4}$ the quotient of the slice disk, and $\bar{B}=D_{\bar{B}} \cap S^{3}$. A Mayer-Vietoris argument shows that $H_{1}\left(\bar{B}^{4}-D_{\bar{B}}-D_{\bar{K}}\right)=\mathbf{Z} \times \mathbf{Z}\left(\right.$ let $\left.\bar{B}^{4}=\left(N\left(D_{\bar{B}}\right) \cup N\left(D_{\bar{K}}\right)\right) \cup\left(\bar{B}^{4}-D_{\bar{B}}-D_{\bar{K}}\right)\right)$. Let $\partial_{+} D^{2}$ and $\partial_{-} D^{2}$ denote the upper and lower semicircles of $S^{1} \subset D^{2}$. Then there are tubular neighborhoods and a map of triads

$$
\begin{gathered}
f:\left(\bar{B}^{4}-N\left(D_{\bar{B}}\right)-N\left(D_{\bar{K}}\right) ; S^{3}-N(\bar{B})-N(\bar{K}), \partial\left(\overline{\left.N\left(D_{\bar{B}}\right) \cup N\left(D_{\bar{K}}\right)\right)}\right)\right. \\
\rightarrow\left(D^{2} \times S^{1} \times S^{1} ; \partial_{+} D^{2} \times S^{1} \times S^{1}, \partial_{-} D^{2} \times S^{1} \times S^{1}\right),
\end{gathered}
$$

inducing a homeomorphism on the third component of the triad.

The proof of Theorem 1.9 follows from Lemma4.2(4) (with $A=S_{e \times \mathbf{Z}}^{-1} \mathbf{Z}[\mathbf{Z} / q \times \mathbf{Z}]$ ), Lemma 4.4(3), and clearing denominators.

Let $K$ be a $q$-equivariant ribbon knot with Alexander polynomial $\Delta(t)$ and Murasugi polynomial $\Delta_{\mathbf{Z} / q}(g, t)$. Since $K$ is equivariant ribbon, $C_{*}(f ; \mathbf{Z}[\mathbf{Z} / q \times \mathbf{Z}])$ is a finite, free $\mathbf{Z}[\mathbf{Z} / q \times \mathbf{Z}]$-chain complex with at most one nonzero homology group $M=H_{2}(f ; \mathbf{Z}[\mathbf{Z} / q \times \mathbf{Z}])$. We claim any such chain complex is chain homotopy equivalent to a finite, free chain complex of the form $D_{3} \rightarrow D_{2}$. The first step is to use that a surjective map to a free module splits, so that $C_{*}(f ; \mathbf{Z}[\mathbf{Z} / q \times \mathbf{Z}])$ is 
chain homotopy equivalent to a finite, free complex $C_{*}^{\prime}$ which is zero below degree 2. Hence $M$ has finite homological dimension, and is $\mathbf{Z}$-free by Poincaré duality, and hence by Rim's theorem [22, Section 4] is projective over $\mathbf{Z}[\mathbf{Z} / q]$. Then we see $M$ is homological dimension 1 , since

$$
0 \rightarrow \mathbf{Z}\left[t, t^{-1}\right] \otimes \mathbf{Z} M \stackrel{\cdot(1-t) \otimes i d}{\longrightarrow} \mathbf{Z}\left[t, t^{-1}\right] \otimes \mathbf{z} M \rightarrow \mathbf{Z} \otimes \mathbf{z} M \rightarrow 0
$$

is a projective $\mathbf{Z}[\mathbf{Z} / q \times \mathbf{Z}]$-resolution of length 1 . If necessary, we may sum on a projective complement to $\mathbf{Z}\left[t, t^{-1}\right] \otimes \mathbf{Z} M \stackrel{\cdot(1-t) \otimes i d}{\longrightarrow} \mathbf{Z}\left[t, t^{-1}\right] \otimes \mathbf{Z} M$ to find an exact sequence

$$
0 \rightarrow D_{3} \rightarrow D_{2} \rightarrow M \rightarrow 0
$$

where $D_{2}$ and $D_{3}$ are finitely generated free over $\mathbf{Z}[\mathbf{Z} / q \times \mathbf{Z}]$. The fundamental lemma of homological algebra then says that $C_{*}^{\prime}$ and $D_{3} \rightarrow D_{2}$ are chain homotopy equivalent.

The existence of a polynomial $a(g, t)$ satisfying the requirements of Theorem 1.10 then follows from Lemma 4.2(4), Lemma 4.4(3), and Lemma 4.1.

Finally, we note that we can use Poincaré duality to give an alternate computation of the Murasugi polynomial of $q$-equivariant ribbon knot that we constructed in Section 3 ,

\section{ACKNOWLEDGEMENT}

The authors would like to thank Charles Livingston for useful conversations throughout this project.

\section{REFERENCES}

[1] I. Aitchison and D. Silver, On certain fibred ribbon disc pairs, Trans. Amer. Math. Soc. 306 (1988) 529-550. MR0933305 (89f:57004)

[2] G. Burde and H. Zieschang, Knots, Studies in Math. 5, de Gruyter, 1985. MR0808776 (87b:57004)

[3] J. C. Cha and K. H. Ko, On equivariant slice knots, Proc. Amer. Math. Soc. 127 (1999) 2175-2182. MR 1605928 (2000a:57006)

[4] J. C. Cha, A characterization of the Murasugi polynomial of an equivariant slice knot, (2004) preprint.

[5] J. Conway, An enumeration of knots and links and some of their algebraic properties in Computational Problems in Abstract Algebra, Proc. Conf. Oxford 1967, 329-358 (ed. John Leech) Pergamon Press, 1970. MR0258014 (41:2661)

[6] J. F. Davis and C. Livingston, Alexander polynomials of periodic knots, Topology 30 (1991) 551-564. MR1133872 (92k:57008)

[7] C. H. Giffen, The generalized Smith conjecture, Amer. J. Math. 88 (1966) 187-198. MR0198462 (33:6620)

[8] R. H. Fox, A quick trip through knot theory, in Topology of 3-Manifolds and Related Topics (Proc. The Univ. of Georgia Institute, 1961), 120-167, (ed. M.K. Fort) Prentice Hall, 1962. MR0140099 (25:3522)

[9] R. H. Fox, Some problems in knot theory, in Topology of 3-Manifolds and Related Topics (Proc. The Univ. of Georgia Institute, 1961), 168-176, (ed. M.K. Fort) Prentice Hall, 1962. MR0140100 (25:3523)

[10] R. H. Fox and J. W. Milnor, Singularities of 2-spheres in 4-space and cobordism of knots, Osaka J. Math. 3 (1966) 257-267. MR0211392 (35:2273)

[11] R. E. Gompf and A. I. Stipsicz, 4-manifolds and Kirby Calculus, Amer. Math. Soc., 1999. MR1707327(2000h:57038)

[12] J. A. Hillman, Polynomials of equivariantly slice knots, (2003) preprint.

[13] R. Kirby, Problems in low dimensional topology, in Geometric Topology (Athens, GA, 1993), 35-473, ed. R. Kirby, Amer. Math. Soc., 1997. MR.1470751 
[14] A. A. Kosinski, Differential Manifolds, Academic Press, 1993. MR1190010 (95b:57001)

[15] K. H. Ko, D. H. Choi, and W. T. Song, Seifert matrices and equivariant concordances of periodic knots, (1997) preprint.

[16] J. Levine, A characterization of knot polynomials, Topology 4 (1965) 135-141. MR0180964 $(31: 5194)$

[17] J. Milnor, A duality theorem for Reidemeister torsion, Ann. of Math. (2) 76 (1962) 137-147. MR0141115 (25:4526)

[18] J. Milnor, Whitehead torsion, Bull. Amer. Math. Soc. 72 (1966) 358-426. MR0196736 $(33: 4922)$

[19] J. Morgan and H. Bass, ed., The Smith Conjecture, Academic Press, 1984. MR 0758459 (86i:57002)

[20] K. Murasugi, On periodic knots, Comment. Math. Helv. 46 (1971) 162-174. MR0292060 $(45: 1148)$

[21] S. Naik, Equivariant slice knots in $S^{3}$, Knots '96 (Tokyo), 81-89, Proceedings of the Fifth International Research Institute of Mathematical Society of Japan, held at Waseda University, Tokyo, July 22-26, 1996, ed. S. Suzuki, World Sci. Publishing, 1997. MR1664952 (99k:57028)

[22] D. S. Rim, Modules over finite groups, Ann. of Math. (2) 69 (1959) 700-712. MR0104721 $(21: 3474)$

[23] D. Rolfsen, Knots and Links, Mathematics Lecture Series, No. 7. Publish or Perish, Inc., 1976. MR0515288 (58:24236)

[24] P. A. Smith, Transformations of finite period, Ann. of Math. (2) 39 (1938) 127-164. MR.1503393

[25] J. R. Smith, Acyclic localizations, J. Pure Appl. Algebra 12 (1978) 117-127. MR0491895 $(58: 11075)$

[26] H. Terasaka On null equivalent knots, Osaka Math. J. 11 (1959) 95-113. MR0117736 $(22: 8511)$

[27] V. G. Turaev, Reidemeister torsion in knot theory, Russian Math. Surveys 41 (1986) 119-182. MR0832411 (87i:57009)

[28] C. T. C. Wall, Surgery on Compact Manifolds, Academic Press, 1970. MR0431216 $(55: 4217)$

[29] G. W. Whitehead, Elements of Homotopy Theory, Springer, 1978. MR 0516508 (80b:55001)

Department of Mathematics, Indiana University, Bloomington, Indiana 47405-4301

Department of Mathematics \& Statistics, University of Nevada, Reno, Nevada 89557 